\title{
Convergent Filter Bases
}

\author{
Roland Coghetto \\ Rue de la Brasserie 5 \\ 7100 La Louvière, Belgium
}

\begin{abstract}
Summary. We are inspired by the work of Henri Cartan [16, Bourbaki 10. (TG. I Filtres) and Claude Wagschal [34. We define the base of filter, image filter, convergent filter bases, limit filter and the filter base of tails (fr: filtre des sections).
\end{abstract}

MSC: 54A20 03B35

Keywords: convergence; filter; filter base; Frechet filter; limit; net; sequence MML identifier: CARDFIL2, version: 8.1.04 5.32.1246

The notation and terminology used in this paper have been introduced in the following articles: 24], [1], 22, [33], 20], [18], 28], 11], [12, [13, [29], 33, [37, [25], [26], 4], [17], 30], [5], 14], 23], [35], 36], [22], 31], 6], 7], [9], 19], [27], and [15].

\section{Filters - Set-Theoretical Approach}

From now on $X$ denotes a non empty set, $\mathcal{F}$ denotes a filter of $X$, and $S$ denotes a family of subsets of $X$.

Let $X$ be a set and $S$ be a family of subsets of $X$. We say that $S$ is upper if and only if

(Def. 1) for every subsets $Y_{1}, Y_{2}$ of $X$ such that $Y_{1} \in S$ and $Y_{1} \subseteq Y_{2}$ holds $Y_{2} \in S$.

Let us note that there exists a $\cap$-closed family of subsets of $X$ which is non empty and there exists a non empty, $\cap$-closed family of subsets of $X$ which is upper.

Let $X$ be a non empty set. Let us note that there exists a non empty, upper, $\cap$-closed family of subsets of $X$ which has non empty elements.

Now we state the propositions: 
(1) $S$ is a non empty, upper, $\cap$-closed family of subsets of $X$ with non empty elements if and only if $S$ is a filter of $X$.

(2) Let us consider non empty sets $X_{1}, X_{2}$, a filter $\mathcal{F}_{1}$ of $X_{1}$, and a filter $\mathcal{F}_{2}$ of $X_{2}$. Then the set of all $f_{1} \times f_{2}$ where $f_{1}$ is an element of $\mathcal{F}_{1}, f_{2}$ is an element of $\mathcal{F}_{2}$ is a non empty family of subsets of $X_{1} \times X_{2}$.

Let $X$ be a non empty set. We say that $X$ is $\cap$-finite closed if and only if

(Def. 2) for every finite, non empty subset $S_{1}$ of $X, \cap S_{1} \in X$.

One can check that there exists a non empty set which is $\cap$-finite closed.

Now we state the proposition:

(3) Let us consider a non empty set $X$. If $X$ is $\cap$-finite closed, then $X$ is ก-closed.

Note that every non empty set which is $\cap$-finite closed is also $\cap$-closed.

(4) Let us consider a set $X$, and a family $S$ of subsets of $X$. Then $S$ is $\cap$-closed and $X \in S$ if and only if $\operatorname{FinMeet} \operatorname{Cl}(S) \subseteq S$.

(5) Let us consider a non empty set $X$, and a non empty subset $A$ of $X$. Then $\{B$, where $B$ is a subset of $X: A \subseteq B\}$ is a filter of $X$.

Let $X$ be a non empty set. Note that every filter of $X$ is $\cap$-closed.

(6) Let us consider a set $X$, and a family $B$ of subsets of $X$. If $B=\{X\}$, then $B$ is upper.

(7) Let us consider a non empty set $X$, and a filter $\mathcal{F}^{\prime}$ of $X$. Then $\mathcal{F}^{\prime} \neq 2^{X}$.

Let $X$ be a non empty set. The functor Filt $(X)$ yielding a non empty set is defined by the term

(Def. 3) the set of all $\mathcal{F}^{\prime}$ where $\mathcal{F}^{\prime}$ is a filter of $X$.

Let $I$ be a non empty set and $M$ be a $(\operatorname{Filt}(X))$-valued many sorted set indexed by $I$. The intersection of the family of filters $M$ yielding a filter of $X$ is defined by the term

(Def. 4) $\operatorname{rng} M$.

Let $\mathcal{F}_{1}, \mathcal{F}_{2}$ be filters of $X$. We say that $\mathcal{F}_{1}$ is coarser than $\mathcal{F}_{2}$ if and only if (Def. 5) $\mathcal{F}_{1} \subseteq \mathcal{F}_{2}$.

One can verify that the predicate is reflexive. We say that $\mathcal{F}_{1}$ is finer than $\mathcal{F}_{2}$ if and only if

(Def. 6) $\mathcal{F}_{2} \subseteq \mathcal{F}_{1}$.

Observe that the predicate is reflexive.

Now we state the propositions:

(8) Let us consider a non empty set $X$, a filter $\mathcal{F}^{\prime}$ of $X$, and a filter $\mathcal{F}$ of $X$. Suppose $\mathcal{F}=\{X\}$. Then $\mathcal{F}$ is coarser than $\mathcal{F}^{\prime}$. 
(9) Let us consider a non empty set $X$, a non empty set $I$, a $($ Filt $(X))$-valued many sorted set $M$ indexed by $I$, an element $i$ of $I$, and a filter $\mathcal{F}^{\prime}$ of $X$. Suppose $\mathcal{F}^{\prime}=M(i)$. Then the intersection of the family of filters $M$ is coarser than $\mathcal{F}^{\prime}$.

(10) Let us consider a set $X$, and a family $S$ of subsets of $X$. Suppose FinMeetCl $(S)$ has non empty elements. Then $S$ has non empty elements.

(11) Let us consider a non empty set $X$, a family $G$ of subsets of $X$, and a filter $\mathcal{F}^{\prime}$ of $X$. Suppose $G \subseteq \mathcal{F}^{\prime}$. Then

(i) $\operatorname{FinMeetCl}(G) \subseteq \mathcal{F}^{\prime}$, and

(ii) FinMeetCl $(G)$ has non empty elements.

The theorem is a consequence of (4).

Let $X$ be a non empty set, $\mathcal{F}^{\prime}$ be a filter of $X$, and $B$ be a non empty subset of $\mathcal{F}^{\prime}$. We say that $B$ is filter basis if and only if

(Def. 7) for every element $f$ of $\mathcal{F}^{\prime}$, there exists an element $b$ of $B$ such that $b \subseteq f$.

Now we state the proposition:

(12) Let us consider a non empty set $X$, a filter $\mathcal{F}^{\prime}$ of $X$, and a non empty subset $B$ of $\mathcal{F}^{\prime}$. Then $\mathcal{F}^{\prime}$ is coarser than $B$ if and only if $B$ is filter basis.

Let $X$ be a non empty set and $\mathcal{F}^{\prime}$ be a filter of $X$. Observe that there exists a non empty subset of $\mathcal{F}^{\prime}$ which is filter basis.

A generalized basis of $\mathcal{F}^{\prime}$ is a filter basis, non empty subset of $\mathcal{F}^{\prime}$. Now we state the proposition:

(13) Let us consider a non empty set $X$. Then every filter of $X$ is a generalized basis of $\mathcal{F}^{\prime}$.

Let $X$ be a set and $B$ be a family of subsets of $X$. The functor $[B]$ yielding a family of subsets of $X$ is defined by

(Def. 8) for every subset $x$ of $X, x \in$ it iff there exists an element $b$ of $B$ such that $b \subseteq x$.

Now we state the propositions:

(14) Let us consider a set $X$, and a family $S$ of subsets of $X$. Then $[S]=$ $\{x$, where $x$ is a subset of $X$ : there exists an element $b$ of $S$ such that $b \subseteq x\}$.

(15) Let us consider a set $X$, and an empty family $B$ of subsets of $X$. Then $[B]=2^{X}$.

(16) Let us consider a set $X$, and a family $B$ of subsets of $X$. If $\emptyset \in B$, then $[B]=2^{X}$. 


\section{Filters - Lattice-Theoretical Approach}

Now we state the propositions:

(17) Let us consider a set $X$, a non empty family $B$ of subsets of $X$, and a subset $L$ of $2_{\subseteq}^{X}$. If $B=L$, then $[B]=\uparrow L$.

(18) Let us consider a set $X$, and a family $B$ of subsets of $X$. Then $B \subseteq[B]$.

Let $X$ be a set and $B_{1}, B_{2}$ be families of subsets of $X$. We say that $B_{1}$ and $B_{2}$ are equivalent generators if and only if

(Def. 9) for every element $b_{1}$ of $B_{1}$, there exists an element $b_{2}$ of $B_{2}$ such that $b_{2} \subseteq b_{1}$ and for every element $b_{2}$ of $B_{2}$, there exists an element $b_{1}$ of $B_{1}$ such that $b_{1} \subseteq b_{2}$.

Let us note that the predicate is reflexive and symmetric.

Let us consider a set $X$ and families $B_{1}, B_{2}$ of subsets of $X$.

Let us assume that $B_{1}$ and $B_{2}$ are equivalent generators. Now we state the propositions:

(19) $\left[B_{1}\right] \subseteq\left[B_{2}\right]$.

(20) $\left[B_{1}\right]=\left[B_{2}\right]$.

Let $X$ be a non empty set, $\mathcal{F}^{\prime}$ be a filter of $X$, and $B$ be a non empty subset of $\mathcal{F}^{\prime}$. The functor $\# B$ yielding a non empty family of subsets of $X$ is defined by the term

(Def. 10) $B$.

Now we state the propositions:

(21) Let us consider a non empty set $X$, a filter $\mathcal{F}^{\prime}$ of $X$, and a generalized basis $B$ of $\mathcal{F}^{\prime}$. Then $\mathcal{F}^{\prime}=[\# B]$.

(22) Let us consider a non empty set $X$, a filter $\mathcal{F}^{\prime}$ of $X$, and a family $B$ of subsets of $X$. If $\mathcal{F}^{\prime}=[B]$, then $B$ is a generalized basis of $\mathcal{F}^{\prime}$.

(23) Let us consider a non empty set $X$, a filter $\mathcal{F}^{\prime}$ of $X$, a generalized basis $B$ of $\mathcal{F}^{\prime}$, a family $S$ of subsets of $X$, and a subset $S_{1}$ of $\mathcal{F}^{\prime}$. Suppose $S=S_{1}$ and $\# B$ and $S$ are equivalent generators. Then $S_{1}$ is a generalized basis of $\mathcal{F}^{\prime}$. The theorem is a consequence of (19), (21), and (22).

(24) Let us consider a non empty set $X$, a filter $\mathcal{F}^{\prime}$ of $X$, and generalized bases $\mathcal{B}_{1}, \mathcal{B}_{2}$ of $\mathcal{F}^{\prime}$. Then $\# \mathcal{B}_{1}$ and $\# \mathcal{B}_{2}$ are equivalent generators. The theorem is a consequence of $(21)$.

Let $X$ be a set and $B$ be a family of subsets of $X$. We say that $B$ is quasi basis if and only if

(Def. 11) for every elements $b_{1}, b_{2}$ of $B$, there exists an element $b$ of $B$ such that $b \subseteq b_{1} \cap b_{2}$. 
Let $X$ be a non empty set. Let us note that there exists a non empty family of subsets of $X$ which is quasi basis and there exists a non empty, quasi basis family of subsets of $X$ which has non empty elements.

A filter base of $X$ is a non empty, quasi basis family of subsets of $X$ with non empty elements. Now we state the proposition:

(25) Let us consider a non empty set $X$, and a filter base $B$ of $X$. Then $[B]$ is a filter of $X$.

Let $X$ be a non empty set and $B$ be a filter base of $X$. The functor $[B)$ yielding a filter of $X$ is defined by the term

(Def. 12) $[B]$.

Now we state the propositions:

(26) Let us consider a non empty set $X$, and filter bases $\mathcal{B}_{1}, \mathcal{B}_{2}$ of $X$. Suppose $\left[\mathcal{B}_{1}\right)=\left[\mathcal{B}_{2}\right)$. Then $\mathcal{B}_{1}$ and $\mathcal{B}_{2}$ are equivalent generators.

(27) Let us consider a non empty set $X$, a filter base $\mathcal{F}$ of $X$, and a filter $\mathcal{F}^{\prime}$ of $X$. Suppose $\mathcal{F} \subseteq \mathcal{F}^{\prime}$. Then $[\mathcal{F})$ is coarser than $\mathcal{F}^{\prime}$.

(28) Let us consider a non empty set $X$, and a family $G$ of subsets of $X$. Suppose FinMeetCl $(G)$ has non empty elements. Then

(i) $\operatorname{FinMeetCl}(G)$ is a filter base of $X$, and

(ii) there exists a filter $\mathcal{F}^{\prime}$ of $X$ such that $\operatorname{FinMeetCl}(G) \subseteq \mathcal{F}^{\prime}$.

The theorem is a consequence of (4).

(29) Let us consider a non empty set $X$, and a filter $\mathcal{F}^{\prime}$ of $X$. Then every generalized basis of $\mathcal{F}^{\prime}$ is a filter base of $X$.

(30) Let us consider a non empty set $X$. Then every filter base of $X$ is a generalized basis of $[B)$.

(31) Let us consider a non empty set $X$, a filter $\mathcal{F}^{\prime}$ of $X$, a generalized basis $B$ of $\mathcal{F}^{\prime}$, and a subset $L$ of $2_{\subset}^{X}$. If $L=\# B$, then $\mathcal{F}^{\prime}=\uparrow L$. The theorem is a consequence of $(21)$ and $(\overline{17})$.

(32) Let us consider a non empty set $X$, a filter base $B$ of $X$, and a subset $L$ of $2_{\subseteq}^{X}$. If $L=B$, then $[B)=\uparrow L$.

(33) Let us consider a non empty set $X$, filters $\mathcal{F}_{1}, \mathcal{F}_{2}$ of $X$, a generalized basis $\mathcal{B}_{1}$ of $\mathcal{F}_{1}$, and a generalized basis $\mathcal{B}_{2}$ of $\mathcal{F}_{2}$. Then $\mathcal{F}_{1}$ is coarser than $\mathcal{F}_{2}$ if and only if $\mathcal{B}_{1}$ is coarser than $\mathcal{B}_{2}$. The theorem is a consequence of (21).

(34) Let us consider non empty sets $X, Y$, a function $f$ from $X$ into $Y$, a filter $\mathcal{F}^{\prime}$ of $X$, and a generalized basis $B$ of $\mathcal{F}^{\prime}$. Then

(i) $f^{\circ}(\# B)$ is a filter base of $Y$, and

(ii) $\left[f^{\circ}(\# B)\right]$ is a filter of $Y$, and 
(iii) $\left[f^{\circ}(\# B)\right]=\left\{M\right.$, where $M$ is a subset of $\left.Y: f^{-1}(M) \in \mathcal{F}^{\prime}\right\}$.

Proof: Set $\mathcal{F}=f^{\circ}(\# B) . \mathcal{F}$ is a quasi basis, non empty family of subsets of $Y$ by (29), [35, (123), (121)]. $\mathcal{F}$ has non empty elements by [35, (118)]. $[\mathcal{F}]=\left\{M\right.$, where $M$ is a subset of $\left.Y: f^{-1}(M) \in \mathcal{F}^{\prime}\right\}$ by [35, (143)], [12, (42)], (21), [35, (123)].

Let $X, Y$ be non empty sets, $f$ be a function from $X$ into $Y$, and $\mathcal{F}^{\prime}$ be a filter of $X$. The image of filter $\mathcal{F}^{\prime}$ under $f$ yielding a filter of $Y$ is defined by the term

(Def. 13) $\left\{M\right.$, where $M$ is a subset of $\left.Y: f^{-1}(M) \in \mathcal{F}^{\prime}\right\}$.

Now we state the propositions:

(35) Let us consider non empty sets $X, Y$, a function $f$ from $X$ into $Y$, and a filter $\mathcal{F}^{\prime}$ of $X$. Then

(i) $f^{\circ} \mathcal{F}^{\prime}$ is a filter base of $Y$, and

(ii) $\left[f^{\circ} \mathcal{F}^{\prime}\right]=$ the image of filter $\mathcal{F}^{\prime}$ under $f$.

The theorem is a consequence of (13) and (34).

(36) Let us consider a non empty set $X$, and a filter base $B$ of $X$. If $B=[B)$, then $B$ is a filter of $X$.

(37) Let us consider non empty sets $X, Y$, a function $f$ from $X$ into $Y$, a filter $\mathcal{F}^{\prime}$ of $X$, and a generalized basis $B$ of $\mathcal{F}^{\prime}$. Then

(i) $f^{\circ}(\# B)$ is a generalized basis of the image of filter $\mathcal{F}^{\prime}$ under $f$, and

(ii) $\left[f^{\circ}(\# B)\right]=$ the image of filter $\mathcal{F}^{\prime}$ under $f$.

The theorem is a consequence of (34) and (30).

(38) Let us consider non empty sets $X, Y$, a function $f$ from $X$ into $Y$, and filter bases $\mathcal{B}_{1}, \mathcal{B}_{2}$ of $X$. Suppose $\mathcal{B}_{1}$ is coarser than $\mathcal{B}_{2}$. Then $\left[\mathcal{B}_{1}\right)$ is coarser than $\left[\mathcal{B}_{2}\right)$. The theorem is a consequence of $(30)$ and $(33)$.

(39) Let us consider non empty sets $X, Y$, a function $f$ from $X$ into $Y$, and a filter $\mathcal{F}^{\prime}$ of $X$. Then $f^{\circ} \mathcal{F}^{\prime}$ is a filter of $Y$ if and only if $Y=\operatorname{rng} f$.

Proof: Reconsider $f_{3}=f^{\circ} \mathcal{F}^{\prime}$ as a filter base of $Y$. $\left[f_{3}\right) \subseteq f_{3}$ by [35, (143)], [11, (76), (77)].

(40) Let us consider a non empty set $X$, a non empty subset $A$ of $X$, a filter $\mathcal{F}^{\prime}$ of $A$, and a generalized basis $B$ of $\mathcal{F}^{\prime}$. Then

(i) $\left(\begin{array}{c}A \\ \hookrightarrow\end{array}\right)^{\circ}(\# B)$ is a filter base of $X$, and

(ii) $\left[(\stackrel{A}{\hookrightarrow})^{\circ}(\# B)\right]$ is a filter of $X$, and

(iii) $\left[\left(\begin{array}{c}A \\ \hookrightarrow\end{array}\right)^{\circ}(\# B)\right]=\left\{M\right.$, where $M$ is a subset of $\left.X:\left(\begin{array}{c}A \\ \hookrightarrow\end{array}\right)^{-1}(M) \in \mathcal{F}^{\prime}\right\}$.

Let $L$ be a non empty relational structure. The functor Tails $(L)$ yielding a non empty family of subsets of $L$ is defined by the term 
(Def. 14) the set of all $\uparrow i$ where $i$ is an element of $L$.

Now we state the proposition:

(41) Let us consider a non empty, transitive, reflexive relational structure $L$. Suppose $\Omega_{L}$ is directed. Then $[\operatorname{Tails}(L)]$ is a filter of $\Omega_{L}$.

Proof: Tails $(L)$ is non empty family of subsets of $L$ and quasi basis and has non empty elements by [6, (22)].

Let $L$ be a non empty, transitive, reflexive relational structure. Assume $\Omega_{L}$ is directed. The functor TailsFilter $L$ yielding a filter of $\Omega_{L}$ is defined by the term

(Def. 15) [Tails $(L)]$.

Now we state the proposition:

(42) Let us consider a non empty, transitive, reflexive relational structure $L$. Suppose $\Omega_{L}$ is directed. Then Tails $(L)$ is a generalized basis of TailsFilter $L$. The theorem is a consequence of (22).

Let $L$ be a relational structure and $x$ be a family of subsets of $L$. The functor $\# x$ yielding a family of subsets of $\Omega_{L}$ is defined by the term

(Def. 16) $x$.

Now we state the proposition:

(43) Let us consider a non empty set $X$, a non empty, transitive, reflexive relational structure $L$, and a function $f$ from $\Omega_{L}$ into $X$. Suppose $\Omega_{L}$ is directed. Then $f^{\circ}(\#$ Tails $(L))$ is a generalized basis of the image of filter TailsFilter $L$ under $f$. The theorem is a consequence of (42) and (37).

Let us consider a non empty set $X$, a non empty, transitive, reflexive relational structure $L$, a function $f$ from $\Omega_{L}$ into $X$, and a subset $x$ of $X$. Now we state the propositions:

(44) Suppose $\Omega_{L}$ is directed and $x \in f^{\circ}(\#$ Tails $(L))$. Then there exists an element $j$ of $L$ such that for every element $i$ of $L$ such that $i \geqslant j$ holds $f(i) \in x$.

(45) Suppose $\Omega_{L}$ is directed and there exists an element $j$ of $L$ such that for every element $i$ of $L$ such that $i \geqslant j$ holds $f(i) \in x$. Then there exists an element $b$ of $\operatorname{Tails}(L)$ such that $f^{\circ} b \subseteq x$.

(46) Let us consider a non empty set $X$, a non empty, transitive, reflexive relational structure $L$, a function $f$ from $\Omega_{L}$ into $X$, a filter $\mathcal{F}^{\prime}$ of $X$, and a generalized basis $B$ of $\mathcal{F}^{\prime}$. Suppose $\Omega_{L}$ is directed. Then $\mathcal{F}^{\prime}$ is coarser than the image of filter TailsFilter $L$ under $f$ if and only if $B$ is coarser than $f^{\circ}(\#$ Tails $(L))$. The theorem is a consequence of (43) and (33).

(47) Let us consider a non empty set $X$, a non empty, transitive, reflexive relational structure $L$, a function $f$ from $\Omega_{L}$ into $X$, and a filter base $B$ of 
$X$. Suppose $\Omega_{L}$ is directed. Then $B$ is coarser than $f^{\circ}(\#$ Tails $(L))$ if and only if for every element $b$ of $B$, there exists an element $i$ of $L$ such that for every element $j$ of $L$ such that $i \leqslant j$ holds $f(j) \in b$. The theorem is a consequence of (44) and (45).

Let $X$ be a non empty set and $s$ be a sequence of $X$. The elementary filter of $s$ yielding a filter of $X$ is defined by the term

(Def. 17) the image of filter FrechetFilter( $\mathbb{N})$ under $s$.

Now we state the propositions:

(48) There exists a sequence $\mathcal{F}^{\prime}$ of $2^{\mathbb{N}}$ such that for every element $x$ of $\mathbb{N}$, $\mathcal{F}^{\prime}(x)=\{y$, where $y$ is an element of $\mathbb{N}: x \leqslant y\}$.

Proof: Define $\mathcal{F}$ (object) $=\{y$, where $y$ is an element of $\mathbb{N}$ : there exists an element $x_{0}$ of $\mathbb{N}$ such that $x_{0}=\$_{1}$ and $\left.x_{0} \leqslant y\right\}$. There exists a function $f$ from $\mathbb{N}$ into $2^{\mathbb{N}}$ such that for every object $x$ such that $x \in \mathbb{N}$ holds $f(x)=\mathcal{F}(x)$ from [12, Sch. 2]. Consider $\mathcal{F}^{\prime}$ being a function from $\mathbb{N}$ into $2^{\mathbb{N}}$ such that for every object $x$ such that $x \in \mathbb{N}$ holds $\mathcal{F}^{\prime}(x)=\mathcal{F}(x)$. For every element $x$ of $\mathbb{N}, \mathcal{F}^{\prime}(x)=\{y$, where $y$ is an element of $\mathbb{N}: x \leqslant y\}$.

(49) Let us consider a natural number $n$. Then $\mathbb{N} \backslash\{t$, where $t$ is an element of $\mathbb{N}: n \leqslant t\}$ is finite.

Proof: $\mathbb{N} \backslash\{t$, where $t$ is an element of $\mathbb{N}: n \leqslant t\} \subseteq n+1$ by [8, (3), (5)], [32, (4)].

(50) Let us consider an element $p$ of the ordered $\mathbb{N}$. Then $\{x$, where $x$ is an element of $\mathbb{N}$ : there exists an element $p_{0}$ of $\mathbb{N}$ such that $p=p_{0}$ and $\left.p_{0} \leqslant x\right\}=\uparrow p$.

Proof: For every element $p$ of the carrier of the ordered $\mathbb{N},\{x$, where $x$ is an element of the carrier of the ordered $\mathbb{N}: p \leqslant x\}=\uparrow p$ by [6, (18)].

Observe that $\Omega_{\text {the ordered } \mathbb{N}}$ is directed and the ordered $\mathbb{N}$ is reflexive.

Now we state the proposition:

(51) Let us consider a denumerable set $X$. Then FrechetFilter $(X)=$ the set of all $X \backslash A$ where $A$ is a finite subset of $X$.

Let us consider a sequence $\mathcal{F}^{\prime}$ of $2^{\mathbb{N}}$.

Let us assume that for every element $x$ of $\mathbb{N}, \mathcal{F}^{\prime}(x)=\{y$, where $y$ is an element of $\mathbb{N}: x \leqslant y\}$. Now we state the propositions:

(52) $\operatorname{rng} \mathcal{F}^{\prime}$ is a generalized basis of FrechetFilter $(\mathbb{N})$.

Proof: FrechetFilter $(\mathbb{N})=$ the set of all $\mathbb{N} \backslash A$ where $A$ is a finite subset of $\mathbb{N}$. For every object $t$ such that $t \in \operatorname{rng} \mathcal{F}^{\prime}$ holds $t \in \operatorname{FrechetFilter}(\mathbb{N})$. Reconsider $\mathcal{F}_{1}=\operatorname{rng} \mathcal{F}^{\prime}$ as a non empty subset of FrechetFilter $(\mathbb{N})$. $\mathcal{F}_{1}$ is filter basis by [21, (2)], [4, (44)], [11, (3)]. 
(53) \# Tails (the ordered $\mathbb{N})=\operatorname{rng} \mathcal{F}^{\prime}$. The theorem is a consequence of $(50)$.

Now we state the proposition:

(54) (i) \# Tails (the ordered $\mathbb{N}$ ) is a generalized basis of FrechetFilter $(\mathbb{N})$, and

(ii) TailsFilter the ordered $\mathbb{N}=\operatorname{FrechetFilter}(\mathbb{N})$.

The theorem is a consequence of (48), (53), (52), and (21).

The base of Frechet filter yielding a filter base of $\mathbb{N}$ is defined by the term

(Def. 18) \# Tails(the ordered $\mathbb{N}$ ).

Now we state the propositions:

(55) $\mathbb{N} \in$ the base of Frechet filter.

(56) The base of Frechet filter is a generalized basis of FrechetFilter $(\mathbb{N})$.

(57) Let us consider a non empty set $X$, filters $\mathcal{F}_{1}, \mathcal{F}_{2}$ of $X$, and a filter $\mathcal{F}^{\prime}$ of $X$. Suppose $\mathcal{F}^{\prime}$ is finer than $\mathcal{F}_{1}$ and $\mathcal{F}^{\prime}$ is finer than $\mathcal{F}_{2}$. Let us consider an element $M_{1}$ of $\mathcal{F}_{1}$, and an element $M_{2}$ of $\mathcal{F}_{2}$. Then $M_{1} \cap M_{2}$ is not empty.

(58) Let us consider a non empty set $X$, and filters $\mathcal{F}_{1}, \mathcal{F}_{2}$ of $X$. Suppose for every element $M_{1}$ of $\mathcal{F}_{1}$ for every element $M_{2}$ of $\mathcal{F}_{2}, M_{1} \cap M_{2}$ is not empty. Then there exists a filter $\mathcal{F}^{\prime}$ of $X$ such that

(i) $\mathcal{F}^{\prime}$ is finer than $\mathcal{F}_{1}$, and

(ii) $\mathcal{F}^{\prime}$ is finer than $\mathcal{F}_{2}$.

Let $X$ be a set and $x$ be a subset of $X$. The functor SubsetToBooleSubset $x$ yielding an element of $2_{\subseteq}^{X}$ is defined by the term

(Def. 19) $x$.

Now we state the propositions:

(59) Let us consider an infinite set $X$. Then $X \in$ the set of all $X \backslash A$ where $A$ is a finite subset of $X$.

(60) Let us consider a set $X$, and a subset $A$ of $X$. Then $\{B$, where $B$ is an element of $\left.2_{\subseteq}^{X}: A \subseteq B\right\}=\{B$, where $B$ is a subset of $X: A \subseteq B\}$.

(61) Let us consider a set $X$, and an element $a$ of $2_{\subseteq}^{X}$. Then $\uparrow a=\{Y$, where $Y$ is a subset of $X: a \subseteq Y\}$.

(62) Let us consider a set $X$, and a subset $A$ of $X$. Then $\{B$, where $B$ is an element of $\left.2_{\subseteq}^{X}: A \subseteq B\right\}=\uparrow$ SubsetToBooleSubset $A$. The theorem is a consequence of $(60)$.

(63) Let us consider a non empty set $X$, and a filter $\mathcal{F}^{\prime}$ of $X$. Then $\cup \mathcal{F}^{\prime}=X$.

(64) Let us consider an infinite set $X$. Then the set of all $X \backslash A$ where $A$ is a finite subset of $X$ is a filter of $X$. The theorem is a consequence of (59). 
Let us consider a set $X$. Now we state the propositions:

(65) $2^{X}$ is a filter of $2_{\subseteq}^{X}$.

(66) $\{X\}$ is a filter of $2_{\subseteq}^{X}$.

(67) Let us consider a non empty set $X$. Then $\{X\}$ is a filter of $X$.

Let us consider an element $A$ of $2_{\subseteq}^{X}$. Now we state the propositions:

(68) $\{Y$, where $Y$ is a subset of $X: A \subseteq Y\}$ is a filter of $2_{\subseteq}^{X}$.

(69) $\left\{B\right.$, where $B$ is an element of $\left.2_{\subset}^{X}: A \subseteq B\right\}$ is a filter of $2_{\subseteq}^{X}$. The theorem is a consequence of (60) and (68).

Now we state the proposition:

(70) Let us consider a non empty set $X$, and a non empty subset $B$ of $2_{\subseteq}^{X}$. Then for every elements $x, y$ of $B$, there exists an element $z$ of $B$ such that $z \subseteq x \cap y$ if and only if $B$ is filtered.

Proof: For every elements $x, y$ of $B$, there exists an element $z$ of $B$ such that $z \subseteq x \cap y$ by [19, (2)].

Let us consider a non empty set $X$ and a non empty subset $\mathcal{F}^{\prime}$ of the lattice of subsets of $X$. Now we state the propositions:

(71) $\mathcal{F}^{\prime}$ is a filter of the lattice of subsets of $X$ if and only if for every elements $p, q$ of $\mathcal{F}^{\prime}, p \cap q \in \mathcal{F}^{\prime}$ and for every element $p$ of $\mathcal{F}^{\prime}$ and for every element $q$ of the lattice of subsets of $X$ such that $p \subseteq q$ holds $q \in \mathcal{F}^{\prime}$.

(72) $\mathcal{F}^{\prime}$ is a filter of the lattice of subsets of $X$ if and only if for every subsets $Y_{1}, Y_{2}$ of $X$, if $Y_{1}, Y_{2} \in \mathcal{F}^{\prime}$, then $Y_{1} \cap Y_{2} \in \mathcal{F}^{\prime}$ and if $Y_{1} \in \mathcal{F}^{\prime}$ and $Y_{1} \subseteq Y_{2}$, then $Y_{2} \in \mathcal{F}^{\prime}$. The theorem is a consequence of (71).

Now we state the propositions:

(73) Let us consider a non empty set $X$, and a non empty family $\mathcal{F}$ of subsets of $X$. Suppose $\mathcal{F}$ is a filter of the lattice of subsets of $X$. Then $\mathcal{F}$ is a filter of $2_{\subseteq}^{X}$. The theorem is a consequence of $(71)$.

(74) Let us consider a non empty set $X$. Then every filter of $2_{\subset}^{X}$ is a filter of the lattice of subsets of $X$. The theorem is a consequence of (72).

(75) Let us consider a non empty set $X$, and a non empty subset $\mathcal{F}^{\prime}$ of the lattice of subsets of $X$. Then $\mathcal{F}^{\prime}$ is filter of the lattice of subsets of $X$ and has non empty elements if and only if $\mathcal{F}^{\prime}$ is a filter of $X$. The theorem is a consequence of (72).

(76) Let us consider a non empty set $X$. Then every proper filter of $2_{\subseteq}^{X}$ is a filter of $X$.

Proof: $\mathcal{F}^{\prime}$ has non empty elements by [19, (18)], [7, (4)].

(77) Let us consider a non empty topological space $T$, and a point $x$ of $T$. Then the neighborhood system of $x$ is a filter of the carrier of $T$. 
Let $T$ be a non empty topological space and $\mathcal{F}^{\prime}$ be a proper filter of $2_{\subseteq}^{\Omega_{T}}$. The functor BooleanFilterToFilter $\left(\mathcal{F}^{\prime}\right)$ yielding a filter of the carrier of $T$ is defined by the term

(Def. 20) $\mathcal{F}^{\prime}$.

Let $\mathcal{F}_{1}$ be a filter of the carrier of $T$ and $\mathcal{F}_{2}$ be a proper filter of $2_{\subseteq}^{\Omega_{T}}$. We say that $\mathcal{F}_{1}$ is finer than $\mathcal{F}_{2}$ if and only if

(Def. 21) BooleanFilterToFilter $\left(\mathcal{F}_{2}\right) \subseteq \mathcal{F}_{1}$.

\section{Limit OF A FiLter}

Let $T$ be a non empty topological space and $\mathcal{F}^{\prime}$ be a filter of the carrier of $T$. The functor LimFilter $\left(\mathcal{F}^{\prime}\right)$ yielding a subset of $T$ is defined by the term

(Def. 22) $\left\{x\right.$, where $x$ is a point of $T: \mathcal{F}^{\prime}$ is finer than the neighborhood system of $x\}$.

Let $B$ be a filter base of the carrier of $T$. The functor $\operatorname{Lim} B$ yielding a subset of $T$ is defined by the term

(Def. 23) LimFilter $([B))$.

Now we state the proposition:

(78) Let us consider a non empty topological space $T$, and a filter $\mathcal{F}^{\prime}$ of the carrier of $T$. Then there exists a proper filter $\mathcal{F}_{1}$ of $2_{\subseteq}^{\alpha}$ such that $\mathcal{F}^{\prime}=\mathcal{F}_{1}$, where $\alpha$ is the carrier of $T$. The theorem is a consequence of (73) and (75).

Let $T$ be a non empty topological space and $\mathcal{F}^{\prime}$ be a filter of the carrier of $T$. The functor FilterToBooleanFilter $\left(\mathcal{F}^{\prime}, T\right)$ yielding a proper filter of $2_{\subseteq}^{\Omega_{T}}$ is defined by the term

(Def. 24) $\mathcal{F}^{\prime}$.

Let us consider a non empty topological space $T$, a point $x$ of $T$, and a filter $\mathcal{F}^{\prime}$ of the carrier of $T$. Now we state the propositions:

(79) $x$ is a convergence point of $\mathcal{F}^{\prime}$ and $T$ if and only if $x$ is a convergence point of FilterToBooleanFilter $\left(\mathcal{F}^{\prime}, T\right)$ and $T$.

(80) $x$ is a convergence point of $\mathcal{F}^{\prime}$ and $T$ if and only if $x \in \operatorname{LimFilter}\left(\mathcal{F}^{\prime}\right)$. The theorem is a consequence of (78).

Let $T$ be a non empty topological space and $\mathcal{F}^{\prime}$ be a filter of $2_{\subseteq}^{\Omega_{T}}$. The functor LimFilterB $\left(\mathcal{F}^{\prime}\right)$ yielding a subset of $T$ is defined by the term

(Def. 25) $\left\{x\right.$, where $x$ is a point of $T$ : the neighborhood system of $\left.x \subseteq \mathcal{F}^{\prime}\right\}$.

Let us consider a non empty topological space $T$ and a filter $\mathcal{F}^{\prime}$ of the carrier of $T$. Now we state the propositions: 
(81) $\operatorname{LimFilter}\left(\mathcal{F}^{\prime}\right)=\operatorname{LimFilterB}\left(\right.$ FilterToBooleanFilter $\left.\left(\mathcal{F}^{\prime}, T\right)\right)$.

(82) $\operatorname{Lim}\left(\right.$ the net of FilterToBooleanFilter $\left.\left(\mathcal{F}^{\prime}, T\right)\right)=\operatorname{LimFilter}\left(\mathcal{F}^{\prime}\right)$.

(83) Let us consider a Hausdorff, non empty topological space $T$, a filter $\mathcal{F}^{\prime}$ of the carrier of $T$, and points $p, q$ of $T$. If $p, q \in \operatorname{LimFilter}\left(\mathcal{F}^{\prime}\right)$, then $p=q$.

Let $T$ be a Hausdorff, non empty topological space and $\mathcal{F}^{\prime}$ be a filter of the carrier of $T$. Note that $\operatorname{LimFilter}\left(\mathcal{F}^{\prime}\right)$ is trivial.

Let $X$ be a non empty set, $T$ be a non empty topological space, $f$ be a function from $X$ into the carrier of $T$, and $\mathcal{F}^{\prime}$ be a filter of $X$. The functor $\lim _{\mathcal{F}^{\prime}} f$ yielding a subset of $\Omega_{T}$ is defined by the term

(Def. 26) LimFilter(the image of filter $\mathcal{F}^{\prime}$ under $f$ ).

Let $L$ be a non empty, transitive, reflexive relational structure and $f$ be a function from $\Omega_{L}$ into the carrier of $T$. The functor $\operatorname{LimF}(f)$ yielding a subset of $\Omega_{T}$ is defined by the term

(Def. 27) LimFilter(the image of filter TailsFilter $L$ under $f$ ).

Now we state the proposition:

(84) Let us consider a non empty topological space $T$, a non empty, transitive, reflexive relational structure $L$, a function $f$ from $\Omega_{L}$ into the carrier of $T$, a point $x$ of $T$, and a generalized basis $B$ of BooleanFilterToFilter(the neighborhood system of $x$ ). Suppose $\Omega_{L}$ is directed. Then $x \in \operatorname{LimF}(f)$ if and only if for every element $b$ of $B$, there exists an element $i$ of $L$ such that for every element $j$ of $L$ such that $i \leqslant j$ holds $f(j) \in b$. The theorem is a consequence of (46), (29), and (47).

Let $T$ be a non empty topological space and $s$ be a sequence of $T$. The functor $\operatorname{LimF}(s)$ yielding a subset of $T$ is defined by the term

(Def. 28) LimFilter(the elementary filter of $s$ ).

Now we state the proposition:

(85) Let us consider a non empty topological space $T$, and a sequence $s$ of $T$. Then $\lim _{\text {FrechetFilter(N) }} s=\operatorname{LimF}(s)$.

Let us consider a non empty topological space $T$ and a point $x$ of $T$.

(86) The neighborhood system of $x$ is a filter base of $\Omega_{T}$. The theorem is a consequence of (76), (13), and (29).

(87) Every generalized basis of BooleanFilterToFilter(the neighborhood system of $x$ ) is a filter base of $\Omega_{T}$.

(88) Let us consider a non empty set $X$, a sequence $s$ of $X$, and a filter base $B$ of $X$. Then $B$ is coarser than $s^{\circ}$ (the base of Frechet filter) if and only if for every element $b$ of $B$, there exists an element $i$ of the ordered $\mathbb{N}$ such that for every element $j$ of the ordered $\mathbb{N}$ such that $i \leqslant j$ holds $s(j) \in b$. 
(89) Let us consider a non empty topological space $T$, a sequence $s$ of $T$, a point $x$ of $T$, and a generalized basis $B$ of BooleanFilterToFilter(the neighborhood system of $x$ ). Then $x \in \lim _{\text {FrechetFilter }(\mathbb{N})} s$ if and only if $B$ is coarser than $s^{\circ}$ (the base of Frechet filter). The theorem is a consequence of (46) and (54).

(90) Let us consider a non empty topological space $T$, a sequence $s$ of $\Omega_{T}$, a point $x$ of $T$, and a generalized basis $B$ of BooleanFilterToFilter(the neighborhood system of $x$ ). Then $B$ is coarser than $s^{\circ}$ (the base of Frechet filter) if and only if for every element $b$ of $B$, there exists an element $i$ of the ordered $\mathbb{N}$ such that for every element $j$ of the ordered $\mathbb{N}$ such that $i \leqslant j$ holds $s(j) \in b$. The theorem is a consequence of (29) and (47).

Let us consider a non empty topological space $T$, a sequence $s$ of the carrier of $T$, a point $x$ of $T$, and a generalized basis $B$ of BooleanFilterToFilter(the neighborhood system of $x$ ).

(91) $x \in \lim _{\text {FrechetFilter(N) }} s$ if and only if for every element $b$ of $B$, there exists an element $i$ of the ordered $\mathbb{N}$ such that for every element $j$ of the ordered $\mathbb{N}$ such that $i \leqslant j$ holds $s(j) \in b$. The theorem is a consequence of (89) and (90).

(92) $x \in \operatorname{LimF}(s)$ if and only if for every element $b$ of $B$, there exists an element $i$ of the ordered $\mathbb{N}$ such that for every element $j$ of the ordered $\mathbb{N}$ such that $i \leqslant j$ holds $s(j) \in b$. The theorem is a consequence of (91).

\section{Nets}

Let $L$ be a 1 -sorted structure and $s$ be a sequence of the carrier of $L$. The net of $s$ yielding a non empty, strict net structure over $L$ is defined by the term

(Def. 29) $\langle\mathbb{N}, \leqslant \mathbb{N}, s\rangle$.

Let $L$ be a non empty 1-sorted structure. Let us note that the net of $s$ is non empty.

Now we state the proposition:

(93) Let us consider a non empty 1-sorted structure $L$, a set $B$, and a sequence $s$ of the carrier of $L$. Then the net of $s$ is eventually in $B$ if and only if there exists an element $i$ of the net of $s$ such that for every element $j$ of the net of $s$ such that $i \leqslant j$ holds (the net of $s)(j) \in B$.

Let us consider a non empty topological space $T$, a sequence $s$ of the carrier of $T$, a point $x$ of $T$, and a generalized basis $B$ of BooleanFilterToFilter(the neighborhood system of $x$ ). Now we state the propositions:

(94) for every element $b$ of $B$, there exists an element $i$ of the ordered $\mathbb{N}$ such that for every element $j$ of the ordered $\mathbb{N}$ such that $i \leqslant j$ holds $s(j) \in b$ if 
and only if for every element $b$ of $B$, there exists an element $i$ of the net of $s$ such that for every element $j$ of the net of $s$ such that $i \leqslant j$ holds (the net of $s)(j) \in b$.

(95) $x \in \operatorname{LimF}(s)$ if and only if for every element $b$ of $B$, the net of $s$ is eventually in $b$. The theorem is a consequence of (92), (94), and (93).

(96) $x \in \operatorname{LimF}(s)$ if and only if for every element $b$ of $B$, there exists an element $i$ of $\mathbb{N}$ such that for every element $j$ of $\mathbb{N}$ such that $i \leqslant j$ holds $s(j) \in b$. The theorem is a consequence of (91).

(97) $x \in \operatorname{LimF}(s)$ if and only if for every element $b$ of $B$, there exists a natural number $i$ such that for every natural number $j$ such that $i \leqslant j$ holds $s(j) \in b$. The theorem is a consequence of (96).

\section{REFERENCES}

[1] Grzegorz Bancerek. Cardinal numbers Formalized Mathematics, 1(2):377-382, 1990.

[2] Grzegorz Bancerek. König's theorem. Formalized Mathematics, 1(3):589-593, 1990.

[3] Grzegorz Bancerek. Complete lattices. Formalized Mathematics, 2(5):719-725, 1991.

[4] Grzegorz Bancerek. The fundamental properties of natural numbers Formalized Mathematics, 1(1):41-46, 1990.

[5] Grzegorz Bancerek. The ordinal numbers. Formalized Mathematics, 1(1):91-96, 1990.

[6] Grzegorz Bancerek. Directed sets, nets, ideals, filters, and maps Formalized Mathematics, 6(1):93-107, 1997.

[7] Grzegorz Bancerek. Prime ideals and filters Formalized Mathematics, 6(2):241-247, 1997.

[8] Grzegorz Bancerek and Krzysztof Hryniewiecki. Segments of natural numbers and finite sequences. Formalized Mathematics, 1(1):107-114, 1990.

[9] Grzegorz Bancerek, Noboru Endou, and Yuji Sakai. On the characterizations of compactness Formalized Mathematics, 9(4):733-738, 2001.

[10] Nicolas Bourbaki. General Topology: Chapters 1-4. Springer Science and Business Media, 2013.

[11] Czesław Byliński. Functions and their basic properties. Formalized Mathematics, 1(1): 55-65, 1990.

[12] Czesław Byliński. Functions from a set to a set Formalized Mathematics, 1(1):153-164, 1990.

[13] Czesław Byliński. Basic functions and operations on functions Formalized Mathematics, $1(\mathbf{1}): 245-254,1990$.

[14] Czesław Byliński. Partial functions. Formalized Mathematics, 1(2):357-367, 1990.

[15] Czesław Byliński. Some basic properties of sets Formalized Mathematics, 1(1):47-53, 1990.

[16] Henri Cartan. Théorie des filtres. C. R. Acad. Sci., CCV:595-598, 1937.

[17] Marek Chmur. The lattice of natural numbers and the sublattice of it. The set of prime numbers, Formalızed Mathematıcs, 2(4):453-459, 1991.

[18] Agata Darmochwał. Finite sets. Formalized Mathematics, 1(1):165-167, 1990.

[19] Adam Grabowski and Robert Milewski. Boolean posets, posets under inclusion and products of relational structures Formalized Mathematıcs, 6(1):117-121, 1997.

[20] Gilbert Lee and Piotr Rudnicki. Dickson's lemma Formalized Mathematics, 10(1):29-37, 2002.

[21] Yatsuka Nakamura and Hisashi Ito. Basic properties and concept of selected subsequence of zero based finite sequences. Formalized Mathematics, 16(3):283-288, 2008. doi: $10.2478 /$ v10037-008-0034-y 
[22] Beata Padlewska. Families of sets Formalized Mathematics, 1(1):147-152, 1990.

[23] Beata Padlewska and Agata Darmochwał. Topological spaces and continuous functions Formalized Mathematics, 1(1):223-230, 1990.

[24] Alexander Yu. Shibakov and Andrzej Trybulec. The Cantor set Formalized Mathematics, 5(2):233-236, 1996.

[25] Andrzej Trybulec. Tuples, projections and Cartesian products Formalized Mathematics, $1(\mathbf{1}): 97-105,1990$.

[26] Andrzej Trybulec. On the sets inhabited by numbers. Formalized Mathematics, 11(4): 341-347, 2003.

[27] Andrzej Trybulec. Moore-Smith convergence Formalized Mathematics, 6(2):213-225, 1997.

[28] Andrzej Trybulec and Agata Darmochwał. Boolean domains Formalized Mathematics, 1 (1):187-190, 1990.

[29] Michał J. Trybulec. Integers Formalized Mathematics, 1(3):501-505, 1990.

[30] Wojciech A. Trybulec and Grzegorz Bancerek. Kuratowski - Zorn lemma Formalized Mathematics, 1(2):387-393, 1990.

[31] Zinaida Trybulec. Properties of subsets Formalized Mathematics, 1(1):67-71, 1990.

[32] Tetsuya Tsunetou, Grzegorz Bancerek, and Yatsuka Nakamura. Zero-based finite sequences Formalized Mathematics, 9(4):825-829, 2001.

[33] Josef Urban. Basic facts about inaccessible and measurable cardinals Formalized Mathematics, 9(2):323-329, 2001.

[34] Claude Wagschal. Topologie et analyse fonctionnelle. Hermann, 1995.

[35] Edmund Woronowicz. Relations and their basic properties. Formalized Mathematics, 1 (1):73-83, 1990.

[36] Edmund Woronowicz. Relations defined on sets. Formalized Mathematics, 1(1):181-186, 1990.

[37] Stanisław Żukowski. Introduction to lattice theory Formalized Mathematics, 1(1):215222, 1990. 